\title{
EVALUATION OF HOTEL QUALITY ATTRIBUTE IMPORTANCE THROUGH FUZZY CORRELATION COEFFICIENT
}

\author{
Olimpia-Iuliana BAN ${ }^{\mathrm{a}}$, Ioan Gheorghe TARA ${ }^{\mathrm{b}}$, Victoria BOGDAN ${ }^{\mathrm{b}}$, \\ Delia TUŞE ${ }^{c}$, Simona Gabriela BOLOGA ${ }^{\mathrm{d}}$ \\ ${ }^{a}$ Department of Economics, University of Oradea, Universității 1, Oradea, Romania \\ ${ }^{b}$ Department of Finance-Accounting, University of Oradea, Universității 1, Oradea, Romania \\ ${ }^{c}$ Department of Mathematics and Informatics, University of Oradea, Universității 1, Oradea, Romania \\ ${ }^{d}$ Faculty of Economics, Agora University of Oradea, Piata Tineretului 8, Oradea, Romania
}

Received 23 October 2015; accepted 11 January 2016

\begin{abstract}
An indirect method of calculation of the importance of attributes as the fuzzy correlation between the performance of attributes and the overall satisfaction was proposed in a recent paper. We apply the method to the results of a survey with respect to the quality of hotel services in Oradea (Romania). Different representations of the answers as triangular fuzzy numbers, as well as distinct analyzes to compare the hierarchies of the attributes with respect to the experience with the hotel and the motivation of the travel are considered.
\end{abstract}

Keywords: triangular fuzzy number, correlation coefficient, importance of attributes, performance of attributes, hotel services, hierarchy of attributes.

JEL Classification: Z33, C02, C44.

\section{Introduction}

Since the human thinking is subjective and ambiguous, the fuzzy numbers are often preferred instead to crisp numbers for modeling in decision making, engineering, science, economy, social sciences and other areas (see e.g. Ban 2011; Ban, A. I., Ban, O. I. 2012; Ban et al. 2015a; Chien, Tsai 2000; Chu, Lin 2009; Deng 2008; Stanojević et al. 2015; Wei 2011; Wei et al. 2012, 2013; Wu et al. 2004; Yeh, Kuo 2003; Zhao et al. 2013). A very good example is the Likert scale, used throughout surveys that are applied. The responses are usually transformed according to the binary logic and the differences between the successive categories are equal even if initially they are not crisp. For example, the set of possible answers \{"strongly disagree", "disagree", "fair", "agree", "strongly agree"\} is transformed into

Corresponding author Olimpia-Iuliana Ban

E-mail: olimpiaban2008@gmail.com 
5 -point Likert scale $\{1 ; 2 ; 3 ; 4 ; 5\}$ even if in this way a quantity of information is lost. That is why it would be more appropriate a modeling of the answers by fuzzy numbers.

The determination of the importance of attributes, as an essential step in many methods related with the decision theory, can be made by direct or indirect methods. Indirect methods were elaborated (see, e.g., Ban et al. 2015a; Deng 2007; Feng et al. 2014; Hair et al. 1995; Hancock, Klockars 1991; Matzler et al. 2003; Mount, Sciarini 1999; Mount 2005) due to the fact that direct methods have significant disadvantages (see, e.g., Abalo et al. 2007; Bacon 2003; Deng, Pei 2009). The input data are often considered as fuzzy numbers such that new methods were proposed. As examples, in Ban et al. (2015a) and Ban et al. (2015b) methods of calculation based on the fuzzy correlation coefficient between the performance of attributes and the overall customer satisfaction were given.

In this paper we apply the method proposed in Ban et al. (2015b) to calculate the importance of attributes in the cases of four 4-star hotels in Oradea, with over 50 rooms and located in high traffic areas. In June 2012, 125 questionnaires were applied to the guests (Romanian and foreign) of these hotels, for measuring the degree of satisfaction regarding the services received, the evaluation of service quality in terms of performance in order to achieve a classification of quality attributes according to the model proposed by Kano (see Ban, Meșter 2014). The data collection instrument was the questionnaire with Likert scale questions to assess the performance of hotel services from the perspective of 21 attributes and to measure the global satisfaction. The results obtained were represented as triangular fuzzy numbers, to shape the subjectivity and ambiguity of human thought. The importance of the attributes was calculated by correlating the overall satisfaction with the perceived performance of each attribute in part, represented by triangular fuzzy numbers, the proposed method being that recently proposed in Ban et al. (2015b).

Our main aim is to evaluate the impact of research variables on the hierarchy of attributes by importance. Based on the recent literature, the following hypotheses are considered in our study:

H1: Due to the small inter-item variation of responses (all respondents give positive values) any drastic penalization of responses which do not represent the maximum on the linguistic scale lead to significant changes in the hierarchy of the importance of attributes.

$\mathrm{H} 2$ : Previous visits and knowledge about the destination play an important role in influencing individual perceptions.

H3: The radical different reasons (e.g. business and leisure) assume different expectations and therefore involve different hierarchies of attributes.

\section{Fuzzy numbers and operations}

We recall that (see Zadeh 1965) a fuzzy set $A$ (fuzzy subset of $X$ is defined as a mapping $A: X \rightarrow[0,1]$, where $A(x)$ is the membership degree of $x$ to the fuzzy set $A$.

The fuzzy numbers generalize the real numbers and they are fuzzy subsets of the real line with some additional properties (see, e.g., Diamond, Kloeden (1994) or Dubois, Prade 
(1978)). In practice fuzzy numbers with simple membership functions are preferred. A triangular fuzzy number (see, e.g., Hong 2006) $\Delta=(a, \alpha, \beta)$ is defined by the membership function:

$$
D(x)=\left\{\begin{array}{cc}
1-\frac{a}{\alpha}+\frac{1}{\alpha} x, \text { if } a-\alpha \leq x \leq a, \\
1+\frac{a}{\beta}-\frac{1}{\beta} x, \text { if } a \leq x \leq a+\beta, \\
0, & \text { otherwise. }
\end{array}\right.
$$

The expected value of a fuzzy number, EV, was introduced in Chanas (2001) and Dubois, Prade (1987). For a triangular fuzzy number $\Delta=(a, \alpha, \beta)$ the general formula becomes:

$$
E V(\Delta)=a+\frac{\beta-\alpha}{4}
$$

In Ban, Coroianu (2015) it was proved that the expected value is a simple and effective ranking index on fuzzy numbers, therefore on triangular fuzzy numbers too. Namely, for two triangular fuzzy numbers $\Delta$ and $\Delta^{\prime}$ we define:

$$
\begin{aligned}
& \Delta \prec \Delta^{\prime} \text { if and only if } E V(\Delta)<E V\left(\Delta^{\prime}\right), \\
& \Delta \sim \Delta^{\prime} \text { if and only if } E V(\Delta)=E V\left(\Delta^{\prime}\right), \\
& \Delta \preceq \Delta^{\prime} \text { if and only if } E V(\Delta) \leq E V\left(\Delta^{\prime}\right) .
\end{aligned}
$$

It is well-known that the Zadeh extension principle based on a triangular norm extends an arithmetical operation on reals to an arithmetical operation on fuzzy numbers (see Hong 2006; Zadeh 1978). If we choose the weakest triangular norm then the operations have some advantages: the calculation is drastically simplified, the fuzziness of the output data is small, the addition and multiplication preserve the shape of fuzzy numbers, in particular of triangular fuzzy numbers, etc. (Hong 2001, 2002).

Below we summarize the arithmetic operations on triangular fuzzy numbers, based on the weakest triangular norm.

Let $\Delta=(a, \alpha, \beta)$ and $\Delta^{\prime}=(b, \gamma, \delta)$ be two triangular fuzzy numbers and $\lambda \in \mathbb{R}, \lambda>0$ we have (see Hong 2006, 2001):

$$
\begin{gathered}
(a, \alpha, \beta)+(b, \gamma, \delta)=(a+b, \max (\alpha, \gamma), \max (\beta, \delta)), \\
(a, \alpha, \beta)-(b, \gamma, \delta)=(a-b, \max (\alpha, \delta), \max (\beta, \gamma)), \\
\lambda \cdot(a, \alpha, \beta)=(\lambda a, \alpha, \beta), \\
(a, \alpha, \beta) \cdot(b, \gamma, \delta)=\left\{\begin{array}{l}
(a b, \max (\alpha b, \gamma a), \max (\beta b, \delta a)), \quad \text { if } a, b \geq 0, \\
(a b,-\max (\alpha b, \gamma a),-\max (\beta b, \delta a)), \text { if } a, b \leq 0, \\
(a b, \max (\alpha b,-\delta a), \max (\beta b,-\gamma a)), \text { if } a \leq 0, b \geq 0, \\
(a b, \max (\gamma a,-\beta b), \max (\delta a,-\alpha b)), \text { if } a \geq 0, b \leq 0 .
\end{array}\right.
\end{gathered}
$$




$$
\begin{aligned}
& \sqrt{(a, \alpha, \beta)}=\left(\sqrt{a}, \frac{\alpha}{\sqrt{a}}, \frac{\beta}{\sqrt{a}}\right), \text { for every } a>0 . \\
& \frac{(a, \alpha, \beta)}{(b, \gamma, \delta)}= \begin{cases}\left(\frac{a}{b}, \max \left(\frac{\alpha}{b}, \frac{a \delta}{b(b+\delta)}\right), \max \left(\frac{\beta}{b}, \frac{a \gamma}{b(b-\gamma)}\right)\right), & \text { if } a>0, b>0, b-\gamma>0, \\
\left(\frac{a}{b}, \max \left(-\frac{\beta}{b},-\frac{a \gamma}{b(b-\gamma)}\right), \max \left(-\frac{\alpha}{b},-\frac{a \delta}{b(b+\delta)}\right)\right), & \text { if } a<0, b<0, b+\delta<0, \\
\left.\left(0,-\frac{\beta}{b},-\frac{\alpha}{b}\right), \frac{\alpha}{b}\right), \operatorname{if} a=0, b>0, b-\gamma>0, & \text { if } a=0, b<0, b+\delta<0, \\
\left(\frac{a}{b}, \max \left(-\frac{\beta}{b}, \frac{a \delta}{b(b+\delta)}\right), \max \left(-\frac{\alpha}{b}, \frac{a \gamma}{b(b-\gamma)}\right)\right), & \text { if } a>0, b<0, b+\delta<0, \\
\left(\frac{a}{b}, \max \left(\frac{\alpha}{b},-\frac{a \gamma}{b(b-\gamma)}\right), \max \left(\frac{\beta}{b},-\frac{a \delta}{b(b+\delta)}\right)\right), & \text { if } a<0, b>0, b-\gamma>0 .\end{cases}
\end{aligned}
$$

\section{Importance of attributes by correlation method under the weakest triangular norm based fuzzy arithmetic}

As in Ban et al. (2015b), let us consider $n$ attributes of a service, $A_{1}, \ldots, A_{n}$, and $m$ customers, consumers of that service, $C_{1}, \ldots, C_{m}$. We denote by $X_{i j}$ the performance of the attribute $A_{j}, j \in\{1, \ldots, n\}$ in the opinion of the customer $C_{i}, i \in\{1, \ldots, m\}$, by $X_{i}$ the overall level of satisfaction in the opinion of the customer $C_{i}, i \in\{1, \ldots, m\}$ and by $W_{i j}$ the importance of the attribute $A_{j}, j \in\{1, \ldots, n\}$ in the opinion of the customer $C_{i}, i \in\{1, \ldots, m\}$. The importance of the attribute $A_{j}$, denoted by $W_{j}$, can be given by a direct method, aggregating the values $W_{i j}, i \in\{1, \ldots, m\}, j \in\{1, \ldots, n\}$. For example, if the arithmetic mean is used, then:

$$
W_{j}=\frac{1}{m} \sum_{i=1}^{m} W_{i j} .
$$

The method can be extended to the fuzzy case in an obvious way by considering the arithmetical operations in (4) and (6). Nevertheless, this method has significant disadvantages (see, e.g., Abalo et al. 2007; Bacon 2003; Deng, Pei 2009).

The correlation coefficient between the performance perceived for each attribute and the overall satisfaction is already accepted as a successful indirect method to determine the importance of the attributes in the crisp case (see Deng 2007; Mount, Sciarini 1999; Mount 2005). Based on the classical definition of the correlation coefficient of two variables (see e.g. Snedecor, Cochran 1967) we obtain that the importance of the attribute $A_{j}, j \in\{1, \ldots, n\}$, is given as the correlation coefficient between $\left(X_{1 j}, \ldots, X_{m j}\right)$ and $\left(X_{1}, \ldots, X_{m}\right)$, therefore: 


$$
W_{j}=\frac{\sum_{i=1}^{m}\left(X_{i j}-X_{j}^{M}\right)\left(X_{i}-X^{M}\right)}{\sqrt{\sum_{i=1}^{m}\left(X_{i j}-X_{j}^{M}\right)^{2} \sum_{i=1}^{m}\left(X_{i}-X^{M}\right)^{2}}},
$$

where $X_{j}^{M}=\frac{1}{m} \sum_{i=1}^{m} X_{i j}$ and $X^{M}=\frac{1}{m} \sum_{i=1}^{m} X_{i}$ The correlation coefficient for two variables expressed by fuzzy numbers was introduced in Liu, Kao (2002). Following the idea in the crisp case we can find the fuzzy importance of attributes as the correlation coefficient between the performance of attributes and the overall satisfaction, all the data being fuzzy numbers (see Ban et al. 2015a).

If the fuzzy number $\tilde{X}_{i j}, i \in\{1, \ldots, m\}, j \in\{1, \ldots, n\}$, denotes the performance of the attribute $A_{j}, j \in\{1, \ldots, n\}$, in the opinion of the customer $C_{i}, i \in\{1, \ldots, m\}$ and the fuzzy number $\tilde{X}_{i}, i \in\{1, \ldots, m\}$, denotes the overall satisfaction in the opinion of the customer $C_{i}$, $i \in\{1, \ldots, m\}$ then we can formally define the fuzzy importance of the attribute $A_{j}, j \in$ $\{1, \ldots, n\}$, as

where:

$$
\widetilde{W}_{j}=\frac{\sum_{i=1}^{m}\left(\tilde{X}_{i j}-\tilde{X}_{j}^{M}\right) \cdot\left(\tilde{X}_{i}-\tilde{X}^{M}\right)}{\sqrt{\sum_{i=1}^{m}\left(\tilde{X}_{i j}-\tilde{X}_{j}^{M}\right)^{2} \sum_{i=1}^{m}\left(\tilde{X}_{i}-\tilde{X}^{M}\right)^{2}}},
$$

and

$$
\tilde{X}_{j}^{M}=\frac{1}{m} \sum_{i=1}^{m} \tilde{X}_{i j}
$$

$$
\tilde{X}^{M}=\frac{1}{m} \sum_{i=1}^{m} \tilde{X}_{i}
$$

In Ban et al. (2015b) $\tilde{X}_{i j}$ and $\tilde{X}_{i}, i \in\{1, \ldots, m\}, j \in\{1, \ldots, n\}$ are considered as triangular fuzzy numbers and the arithmetical operations are given by (4)-(9) such that an analytical result for the fuzzy importance of an attribute is obtained. An algorithm for calculating the importance of the attributes in the triangular fuzzy case was elaborated in Ban et al. (2015b) too. Moreover, the fuzzy numbers obtained from (10)-(12) are ordered by the method described in (1)-(3).

\section{Study of the hotel services in Oradea}

In this section we apply the method already proposed in Ban et al. (2015b) and summarized in the previous section to the study of the quality of hotel services. The same survey as in Ban et al. (2015a, 2015b) is considered, but the study is more detailed.

Although widely used, the Likert scale has several disadvantages (see Dolnicar 2007; Hancock, Klockars 1991; Preston, Colman 2000; Watson 1992), among these being the concentration of responses only at the top of the scale, thus not making a clear and useful enough distinction among the possible answers. This is why we propose a comparative analysis of the hierarchy of quality attributes according to their importance, in two distinct situations: when using the responses on a classic five-steps symmetrical Likert scale and 
when using an asymmetrical Likert scale, which penalizes drastically the answers that are not in the positive extremity, to counteract the disadvantages described above and to force the differentiation. The responses are represented by triangular fuzzy numbers and then the method in Ban et al. (2015b) is applied to obtain values of the importance of attributes, given also by triangular fuzzy numbers. A real number to represent the importance of each attribute and, therefore, a hierarchy of attributes for both approaches are provided.

On the other hand, there have been distinct analyzes, based also on the methods given in Ban et al. (2015b), to compare the hierarchies of the attributes for those who are for the first time at a certain hotel and for those who are not accommodated for the first time at the hotel. Also, based on the same type of approach, we obtained results related to the motivation of the travel, being considered the most important categories: business and pleasure.

During two weeks in June 2012 a number of 125 questionnaires was applied to customers of four 4-stars hotels from Oradea, Romania. For the establishment of the attributes the SERVQUAL scale was considered. The complete list of attributes was included in Table 1.

Table 1. List of attributes

\begin{tabular}{ll}
\hline 1 & The room facilities are appropriate \\
\hline 2 & The room is clean enough \\
\hline 3 & The hotel has sufficient restaurant facilities \\
\hline 4 & The staff has an appropriate and professional look \\
\hline 5 & The location of the hotel is suitable \\
\hline 7 & The staff provide correct information to guests \\
\hline 8 & The staff is able to offer services in a short period of time \\
\hline 9 & The staff is able to provide information in a short time \\
\hline 10 & The availability of staff \\
\hline 11 & Clients complaints are resolved quickly \\
\hline 12 & Different payment facilities are available \\
\hline 13 & The safety of the installations in the hotel \\
\hline 14 & Service professionalism \\
\hline 15 & Service customization \\
\hline 16 & Friendliness of staff \\
\hline 17 & Proper opening hours of hotel's facilities \\
\hline 18 & The hotel has entertaining facilities \\
\hline 19 & Big variety and proper quality of meals \\
\hline 20 & Internet connection is available \\
\hline 21 & Aesthetics of rooms and of the hotel \\
\hline
\end{tabular}

We obtained the value of the $\alpha$-Cronbach coefficient (0:827) as being a satisfactory one (see Ban, Meşter 2014) for the validity of the questionnaire. The performance of attributes and the overall customer satisfaction (OCS) measured on a five Likert scale \{Very poor $(V P)$, Poor (P), Medium (M), Good (G), Very good (VG)\} for all hotels and separately for each hotel are summarized in Table 2. 


\subsection{Symmetric case versus drastic case}

Usually, the distinguishability between $V G$ and $G$ is the same as between $V P$ and $P$, between $G$ and $F$ is the same as between $P$ and $F$, therefore a symmetric case is considered. We can attach the triangular fuzzy numbers to linguistic variables as they are indicated in Table 3.

Table 2. Performance of attributes

\begin{tabular}{|c|c|c|c|c|c|c|c|c|c|c|c|c|c|c|c|c|c|c|c|c|c|c|c|}
\hline & \multicolumn{23}{|c|}{ Attribute } \\
\hline \multirow[t]{3}{*}{ Hotel } & & 1 & 2 & 3 & 4 & 5 & 6 & 7 & 8 & 9 & 10 & 11 & 12 & 13 & 14 & 15 & 16 & 17 & 18 & 19 & 29 & 21 & OCS \\
\hline & $\mathrm{VP}$ & 0 & 0 & 0 & 0 & 0 & 0 & 0 & 0 & 0 & 0 & 0 & 0 & 0 & 0 & 0 & 0 & 0 & 0 & 0 & 0 & 0 & 0 \\
\hline & $\mathrm{P}$ & 2 & 1 & 1 & 2 & 3 & 2 & 0 & 2 & 2 & 2 & 3 & 2 & 1 & 2 & 1 & 2 & 2 & 4 & 2 & 2 & 0 & 0 \\
\hline \multirow[t]{5}{*}{ All } & $M$ & 8 & 9 & 10 & 5 & 15 & 11 & 13 & 13 & 13 & 10 & 10 & 13 & 9 & 10 & 16 & 14 & 12 & 13 & 10 & 15 & 10 & 9 \\
\hline & G & 43 & 33 & 49 & 46 & 49 & 35 & 29 & 41 & 53 & 42 & 46 & 35 & 43 & 48 & 57 & 42 & 49 & 42 & 61 & 39 & 29 & 45 \\
\hline & VG & 72 & 82 & 65 & 72 & 58 & 77 & 83 & 69 & 57 & 71 & 66 & 75 & 72 & 65 & 51 & 67 & 62 & 66 & 52 & 69 & 86 & 71 \\
\hline & $\mathrm{VP}$ & 0 & 0 & 0 & 0 & 0 & 0 & 0 & 0 & 0 & 0 & 0 & 0 & 0 & 0 & 0 & 0 & 0 & 0 & 0 & 0 & 0 & 0 \\
\hline & $\mathrm{P}$ & 0 & 0 & 0 & 0 & 0 & 0 & 0 & 0 & 0 & 0 & 0 & 0 & 0 & 0 & 0 & 0 & 0 & 0 & 0 & 2 & 0 & 0 \\
\hline \multirow[t]{5}{*}{ Hotel 1} & $\mathrm{M}$ & 1 & 1 & 3 & 0 & 1 & 1 & 4 & 3 & 2 & 1 & 4 & 2 & 2 & 1 & 3 & 1 & 1 & 1 & 2 & 5 & 0 & 1 \\
\hline & G & 7 & 0 & 16 & 7 & 13 & 3 & 5 & 5 & 11 & 6 & 9 & 13 & 10 & 8 & 17 & 2 & 15 & 13 & & 8 & 6 & 6 \\
\hline & VG & 31 & 38 & 20 & 32 & 25 & 35 & 30 & 31 & 26 & 32 & 26 & 24 & 27 & 30 & 19 & 36 & 23 & 25 & 12 & 24 & 33 & 32 \\
\hline & $\mathrm{VP}$ & 0 & 0 & 0 & 0 & 0 & 0 & 0 & 0 & 0 & 0 & 0 & 0 & 0 & 0 & 0 & 0 & 0 & 0 & 0 & 0 & 0 & 0 \\
\hline & $\mathrm{P}$ & 0 & 0 & 0 & 0 & 0 & 0 & 0 & 0 & 0 & 0 & 0 & 0 & 0 & 0 & 0 & 0 & 0 & 0 & 0 & 0 & 0 & 0 \\
\hline \multirow[t]{5}{*}{ Hotel 2} & $\mathrm{M}$ & 0 & 1 & 0 & 0 & 2 & 0 & 0 & 1 & 1 & 1 & 0 & 1 & 0 & 0 & 1 & 1 & 0 & 0 & 1 & 0 & 0 & 1 \\
\hline & $\mathrm{G}$ & 1 & 1 & 3 & 6 & 7 & 2 & 3 & 3 & 4 & 2 & 4 & 2 & 2 & 3 & 6 & 3 & 5 & 3 & 2 & 2 & 1 & 2 \\
\hline & VG & 20 & 19 & 18 & 15 & 12 & 19 & 18 & 17 & 16 & 18 & 17 & 18 & 19 & 18 & 14 & 17 & 16 & 18 & 18 & 19 & 20 & 18 \\
\hline & $\mathrm{VP}$ & 0 & 0 & 0 & 0 & 0 & 0 & 0 & 0 & 0 & 0 & 0 & 0 & 0 & 0 & 0 & 0 & 0 & 0 & 0 & 0 & 0 & 0 \\
\hline & $\mathrm{P}$ & 2 & 1 & 1 & 2 & 3 & 2 & 0 & 2 & 2 & 2 & 3 & 2 & 1 & 2 & 1 & 2 & 2 & 4 & 2 & 0 & 0 & 0 \\
\hline \multirow[t]{5}{*}{ Hotel 3} & M & 7 & 7 & 7 & 5 & 7 & 9 & 8 & 9 & 9 & 8 & 4 & 8 & 6 & 8 & 10 & 9 & 8 & 10 & 5 & 8 & 6 & 4 \\
\hline & $\mathrm{G}$ & 14 & 18 & 11 & 13 & 11 & 12 & 12 & 11 & 15 & 13 & 16 & 6 & 14 & 12 & 15 & 17 & 9 & 9 & 15 & 13 & 14 & 21 \\
\hline & VG & 6 & 3 & 10 & 9 & 8 & 6 & 9 & 7 & 3 & 6 & 6 & 13 & 8 & 7 & 3 & 1 & 10 & 6 & 7 & 8 & 9 & 4 \\
\hline & $\mathrm{VP}$ & 0 & 0 & 0 & 0 & 0 & 0 & 0 & 0 & 0 & 0 & 0 & 0 & 0 & 0 & 0 & 0 & 0 & 0 & 0 & 0 & 0 & 0 \\
\hline & $\mathrm{P}$ & 0 & 0 & 0 & 0 & 0 & 0 & 0 & 0 & 0 & 0 & 0 & 0 & 0 & 0 & 0 & 0 & 0 & 0 & 0 & 0 & 0 & 0 \\
\hline \multirow[t]{3}{*}{ Hotel 4} & $\mathrm{M}$ & 0 & 0 & 0 & 0 & 5 & 1 & 1 & 0 & 1 & 0 & 2 & 2 & 1 & 1 & 2 & 3 & 3 & 2 & 2 & 2 & 4 & 3 \\
\hline & $\mathrm{G}$ & 21 & 14 & 19 & 20 & 18 & 18 & 9 & 22 & 23 & 21 & 17 & 14 & 17 & 25 & 19 & 20 & 20 & 17 & 19 & 16 & 8 & 16 \\
\hline & VG & 15 & 22 & 17 & 16 & 13 & 17 & 26 & 14 & 12 & 15 & 17 & 20 & 18 & 10 & 15 & 13 & 13 & 17 & 15 & 18 & 24 & 17 \\
\hline
\end{tabular}

On the other hand, to the question on the importance attributed to the quality characteristics, all respondents gave positive values, very high and very close, suggesting that the characteristics have great and almost similar importance. This small inter-item variation is a known problem of direct research (see Bacon 2003; Deng, Pei 2009). We can try to counteract this trend by drastically penalizing any response that does not represent the maximum on the linguistic scale considered. We modify the combination of Table 3 and we suggest the representation by triangular fuzzy numbers of the linguistic variables as in Table 4 . 
Table 3. Linguistic variables-symmetric case

\begin{tabular}{cc}
\hline Performance/OCS & Triangular fuzzy number \\
\hline Very poor $(\mathrm{VP})$ & $(1,1,1)$ \\
\hline Poor $(\mathrm{P})$ & $(3,2,1)$ \\
\hline Medium $(\mathrm{M})$ & $(5,2,2)$ \\
\hline Good $(\mathrm{G})$ & $(7,1,2)$ \\
\hline Very good $(\mathrm{VG})$ & $(9,1,1)$ \\
\hline
\end{tabular}

Table 4. Linguistic variables-drastic case

\begin{tabular}{cc}
\hline OSC/Performance & Triangular fuzzy number \\
\hline Very poor $(\mathrm{VP})$ & $(0,0,1)$ \\
\hline Poor $(\mathrm{P})$ & $(1,1,1)$ \\
\hline Medium $(\mathrm{M})$ & $(2,1,1)$ \\
\hline Good $(\mathrm{G})$ & $(4,1,1)$ \\
\hline Very good $(\mathrm{VG})$ & $(9,1,1)$ \\
\hline
\end{tabular}

We apply the method described in Section 2 to the data in Table 2 for all hotels and separately for each hotel, in the symmetric case and in the drastic case. We obtain the fuzzy importance of attributes in Tables 5-9. We give the importance of attributes expressed by real numbers after defuzzification with the expected value in the same tables.

Taking into account the real values of the importance of attributes and the ranking defined by (1)-(3) in Table 10 we give the decreasing orders of the importance of attributes for all hotels and separately for each hotel in the symmetric and drastic case.

Table 5. Fuzzy importance and crisp importance of attributes - All hotels

\begin{tabular}{|c|c|c|c|c|}
\hline \multirow{2}{*}{$\begin{array}{l}\text { All hotels } \\
\text { Attribute } \\
\end{array}$} & \multicolumn{2}{|c|}{ Symmetric } & \multicolumn{2}{|l|}{ Drastic } \\
\hline & Fuzzy imp. & Crisp imp. & Fuzzy imp. & Crisp imp. \\
\hline 1 & $(0.30,0.05,0.05)$ & 0.301 & $(0.27,0.01,0.01)$ & 0.270 \\
\hline 2 & $(0.42,0.05,0.05)$ & 0.420 & $(0.43,0.01,0.01)$ & 0.433 \\
\hline 3 & $(0.35,0.03,0.03)$ & 0.355 & $(0.31,0.01,0.01)$ & 0.309 \\
\hline 4 & $(0.42,0.03,0.03)$ & 0.425 & $(0.32,0.01,0.01)$ & 0.315 \\
\hline 5 & $(0.36,0.02,0.02)$ & 0.358 & $(0.29,0.00,0.00)$ & 0.287 \\
\hline 6 & $(0.41,0.03,0.03)$ & 0.410 & $(0.39,0.01,0.01)$ & 0.387 \\
\hline 7 & $(0.19,0.03,0.03)$ & 0.194 & $(0.20,0.01,0.01)$ & 0.203 \\
\hline 8 & $(0.42,0.03,0.03)$ & 0.417 & $(0.38,0.00,0.00)$ & 0.376 \\
\hline 9 & $(0.44,0.04,0.04)$ & 0.444 & $(0.44,0.01,0.01)$ & 0.442 \\
\hline 10 & $(0.39,0.03,0.03)$ & 0.390 & $(0.33,0.01,0.01)$ & 0.330 \\
\hline 11 & $(0.31,0.04,0.04)$ & 0.315 & $(0.34,0.01,0.01)$ & 0.342 \\
\hline 12 & $(0.18,0.03,0.03)$ & 0.175 & $(0.16,0.00,0.00)$ & 0.158 \\
\hline 13 & $(0.24,0.03,0.03)$ & 0.244 & $(0.25,0.01,0.01)$ & 0.245 \\
\hline 14 & $(0.37,0.03,0.03)$ & 0.374 & $(0.37,0.01,0.01)$ & 0.370 \\
\hline 15 & $(0.21,0.03,0.03)$ & 0.210 & $(0.21,0.01,0.01)$ & 0.215 \\
\hline 16 & $(0.37,0.03,0.03)$ & 0.370 & $(0.40,0.00,0.00)$ & 0.402 \\
\hline 17 & $(0.34,0.03,0.03)$ & 0.339 & $(0.33,0.00,0.00)$ & 0.333 \\
\hline 18 & $(0.32,0.04,0.04)$ & 0.316 & $(0.31,0.01,0.01)$ & 0.311 \\
\hline 19 & $(0.17,0.04,0.04)$ & 0.171 & $(0.15,0.01,0.01)$ & 0.151 \\
\hline 20 & $(0.10,0.04,0.04)$ & 0.104 & $(0.16,0.01,0.01)$ & 0.161 \\
\hline 21 & $(0.25,0.03,0.03)$ & 0.248 & $(0.25,0.01,0.01)$ & 0.255 \\
\hline
\end{tabular}


Comparing the hierarchy of attributes obtained by considering a symmetrical scale with the hierarchy of the attributes resulted by considering a drastic scale, we note the following: of the 21 attributes considered, 8 are positioned the same in the two cases, in the last 8 positions. This may suggest that less important attributes are constant and maintain their position regardless of the constraints applied on the answers. At the other extreme, on the top 5 positions are 4 features that retain their position, suggesting that the highly important considered attributes remain so no matter the constraints applied on responses. We should note however that the attributes considered important which retain their position are less in number (half) than those not important which retain their position. Surprisingly, at the first three hotels the situation is similar as a whole. The exception is Hotel 4 where the ratio is reversed, meaning there are more important considered attributes that retain their position than those considered unimportant which retain their position. The conclusion would be that the scale is not that important as suggested (see Hancock, Klockars 1991).

Table 6. Fuzzy importance and crisp importance of attributes - Hotel 1

\begin{tabular}{|c|c|c|c|c|}
\hline Hotel 1 & Symmetric & & Drastic & \\
\hline Attribute & Fuzzy imp. & Crisp imp. & Fuzzy imp. & Crisp imp. \\
\hline 1 & $(-0.21,0.21,0.21)$ & -0.214 & $(-0.23,0.04,0.04)$ & -0.233 \\
\hline 2 & $(-0.07,0.34,0.34)$ & -0.072 & $(-0.08,0.08,0.08)$ & -0.075 \\
\hline 3 & $(0.39,0.13,0.13)$ & 0.393 & $(0.29,0.02,0.02)$ & 0.292 \\
\hline 4 & $(0.23,0.12,0.12)$ & 0.226 & $(0.17,0.03,0.03)$ & 0.175 \\
\hline 5 & $(0.10,0.17,0.17)$ & 0.095 & $(0.08,0.03,0.03)$ & 0.084 \\
\hline 6 & $(-0.14,0.26,0.26)$ & -0.141 & $(-0.15,0.05,0.05)$ & -0.154 \\
\hline 7 & $(-0.06,0.15,0.15)$ & -0.056 & $(0.00,0.03,0.03)$ & -0.001 \\
\hline 8 & $(0.16,0.17,0.17)$ & 0.162 & $(0.22,0.03,0.03)$ & 0.219 \\
\hline 9 & $(0.18,0.15,0.15)$ & 0.183 & $(0.22,0.03,0.03)$ & 0.220 \\
\hline 10 & $(-0.08,0.21,0.21)$ & -0.077 & $(-0.06,0.04,0.04)$ & -0.062 \\
\hline 11 & $(0.29,0.13,0.13)$ & 0.290 & $(0.35,0.02,0.02)$ & 0.351 \\
\hline 12 & $(-0.14,0.17,0.17)$ & -0.140 & $(-0.12,0.03,0.03)$ & -0.120 \\
\hline 13 & $(-0.18,0.17,0.17)$ & -0.180 & $(-0.18,0.03,0.03)$ & -0.179 \\
\hline 14 & $(0.11,0.20,0.20)$ & 0.107 & $(0.17,0.03,0.03)$ & 0.171 \\
\hline 15 & $(-0.24,0.16,0.16)$ & -0.239 & $(-0.23,0.03,0.03)$ & -0.234 \\
\hline 16 & $(-0.12,0.28,0.28)$ & -0.120 & $(-0.13,0.05,0.05)$ & -0.132 \\
\hline 17 & $(0.05,0.18,0.18)$ & 0.052 & $(0.11,0.03,0.03)$ & 0.109 \\
\hline 18 & $(0.10,0.19,0.19)$ & 0.095 & $(0.16,0.03,0.03)$ & 0.159 \\
\hline 19 & $(0.11,0.13,0.13)$ & 0.108 & $(0.07,0.02,0.02)$ & 0.068 \\
\hline 20 & $(-0.24,0.15,0.15)$ & -0.243 & $(-0.25,0.02,0.02)$ & -0.252 \\
\hline 21 & $(-0.19,0.14,0.28)$ & -0.154 & $(-0.20,0.04,0.04)$ & -0.197 \\
\hline
\end{tabular}


Table 7. Fuzzy importance and crisp importance of attributes - Hotel 2

\begin{tabular}{|c|c|c|c|c|}
\hline Hotel 2 & Symmetric & & Drastic & \\
\hline Attribute & Fuzzy imp. & Crisp imp. & Fuzzy imp. & Crisp imp. \\
\hline 1 & $(0.81,0.43,0.43)$ & 0.810 & $(0.69,0.11,0.17)$ & 0.701 \\
\hline 2 & $(0.70,0.37,0.48)$ & 0.728 & $(0.52,0.08,0.08)$ & 0.519 \\
\hline 3 & $(0.66,0.23,0.35)$ & 0.691 & $(0.65,0.06,0.06)$ & 0.647 \\
\hline 4 & $(0.39,0.15,0.21)$ & 0.406 & $(0.37,0.04,0.04)$ & 0.374 \\
\hline 5 & $(0.27,0.21,0.21)$ & 0.274 & $(0.26,0.04,0.04)$ & 0.261 \\
\hline 6 & $(0.20,0.29,0.59)$ & 0.275 & $(0.27,0.10,0.10)$ & 0.272 \\
\hline 7 & $(0.39,0.23,0.23)$ & 0.389 & $(0.31,0.06,0.06)$ & 0.309 \\
\hline 8 & $(0.55,0.32,0.32)$ & 0.552 & $(0.34,0.06,0.06)$ & 0.336 \\
\hline 9 & $(0.50,0.30,0.30)$ & 0.498 & $(0.28,0.06,0.06)$ & 0.275 \\
\hline 10 & $(0.62,0.35,0.35)$ & 0.618 & $(0.41,0.07,0.07)$ & 0.413 \\
\hline 11 & $(0.30,0.20,0.20)$ & 0.301 & $(0.23,0.05,0.05)$ & 0.226 \\
\hline 12 & $(0.43,0.35,0.35)$ & 0.427 & $(0.54,0.07,0.07)$ & 0.542 \\
\hline 13 & $(0.20,0.29,0.59)$ & 0.275 & $(0.27,0.10,0.10)$ & 0.272 \\
\hline 14 & $(0.39,0.25,0.49)$ & 0.451 & $(0.51,0.08,0.08)$ & 0.511 \\
\hline 15 & $(0.24,0.27,0.27)$ & 0.245 & $(0.28,0.05,0.05)$ & 0.281 \\
\hline 16 & $(0.55,0.15,0.29)$ & 0.589 & $(0.56,0.05,0.05)$ & 0.563 \\
\hline 17 & $(0.01,0.20,0.41)$ & 0.061 & $(0.05,0.07,0.07)$ & 0.053 \\
\hline 18 & $(0.39,0.23,0.23)$ & 0.389 & $(0.31,0.06,0.06)$ & 0.309 \\
\hline 19 & $(0.62,0.35,0.35)$ & 0.618 & $(0.41,0.07,0.07)$ & 0.413 \\
\hline 20 & $(0.53,0.29,0.53)$ & 0.584 & $(0.43,0.07,0.07)$ & 0.433 \\
\hline 21 & $(-0.09,0.43,0.81)$ & 0.011 & $(-0.09,0.14,0.14)$ & -0.090 \\
\hline
\end{tabular}

Table 8. Fuzzy importance and crisp importance of attributes - Hotel 3

\begin{tabular}{ccccc}
\hline Hotel 3 & \multicolumn{2}{c}{ Symmetric } & Drastic & \\
\hline Attribute & Fuzzy imp. & Crisp imp. & Fuzzy imp. & Crisp imp. \\
\hline 1 & $(0.16,0.14,0.14)$ & 0.158 & $(0.07,0.03,0.03)$ & 0.067 \\
\hline 2 & $(0.20,0.18,0.18)$ & 0.198 & $(0.20,0.05,0.05)$ & 0.205 \\
\hline 3 & $(0.39,0.08,0.08)$ & 0.386 & $(0.37,0.03,0.03)$ & 0.369 \\
\hline 4 & $(0.45,0.08,0.08)$ & 0.452 & $(0.05,0.03,0.03)$ & 0.050 \\
\hline 5 & $(0.41,0.08,0.08)$ & 0.415 & $(0.17,0.03,0.03)$ & 0.170 \\
\hline 6 & $(0.23,0.10,0.10)$ & 0.230 & $(0.01,0.03,0.03)$ & 0.010 \\
\hline 7 & $(0.09,0.09,0.09)$ & 0.086 & $(0.01,0.03,0.03)$ & 0.006 \\
\hline 8 & $(0.30,0.09,0.09)$ & 0.296 & $(0.15,0.03,0.03)$ & 0.155 \\
\hline 9 & $(0.09,0.14,0.14)$ & 0.087 & $(-0.06,0.04,0.04)$ & -0.061 \\
\hline 10 & $(0.31,0.09,0.09)$ & 0.310 & $(0.12,0.03,0.03)$ & 0.119 \\
\hline 11 & $(0.00,0.14,0.14)$ & 0.000 & $(0.23,0.05,0.05)$ & 0.226 \\
\hline
\end{tabular}


End of Table 8

\begin{tabular}{ccccc}
\hline Hotel 3 & \multicolumn{2}{c}{ Symmetric } & Drastic & \\
\hline Attribute & Fuzzy imp. & Crisp imp. & Fuzzy imp. & Crisp imp. \\
\hline 12 & $(0.00,0.07,0.07)$ & 0.000 & $(0.54,0.07,0.07)$ & 0.542 \\
\hline 13 & $(0.25,0.08,0.08)$ & 0.250 & $(0.27,0.10,0.10)$ & 0.272 \\
\hline 14 & $(0.23,0.09,0.09)$ & 0.225 & $(0.51,0.08,0.08)$ & 0.511 \\
\hline 15 & $(0.09,0.12,0.12)$ & 0.094 & $(0.28,0.05,0.05)$ & 0.281 \\
\hline 16 & $(0.00,0.14,0.14)$ & 0.000 & $(0.56,0.05,0.05)$ & 0.563 \\
\hline 17 & $(0.28,0.07,0.07)$ & 0.278 & $(0.05,0.07,0.07)$ & 0.053 \\
\hline 18 & $(0.07,0.11,0.11)$ & 0.068 & $(0.31,0.06,0.06)$ & 0.309 \\
\hline 19 & $(-0.16,0.15,0.15)$ & -0.159 & $(0.41,0.07,0.07)$ & 0.413 \\
\hline 20 & $(0.09,0.09,0.09)$ & 0.088 & $(0.43,0.07,0.07)$ & 0.433 \\
\hline 21 & $(0.37,0.09,0.09)$ & 0.369 & $(-0.09,0.14,0.14)$ & -0.090 \\
\hline
\end{tabular}

Table 9. Fuzzy importance and crisp importance of attributes - Hotel 4

\begin{tabular}{|c|c|c|c|c|}
\hline \multirow{2}{*}{$\begin{array}{l}\text { Hotel } 4 \\
\text { Attribute }\end{array}$} & \multicolumn{2}{|c|}{ Symmetric } & \multicolumn{2}{|l|}{ Drastic } \\
\hline & Fuzzy imp. & Crisp imp. & Fuzzy imp. & Crisp imp. \\
\hline 1 & $(-0.16,0.06,0.12)$ & -0.147 & $(-0.21,0.02,0.02)$ & -0.206 \\
\hline 2 & $(-0.05,0.06,0.12)$ & -0.034 & $(-0.05,0.02,0.02)$ & -0.048 \\
\hline 3 & $(-0.14,0.06,0.12)$ & -0.126 & $(-0.13,0.02,0.02)$ & -0.130 \\
\hline 4 & $(-0.02,0.06,0.12)$ & -0.004 & $(-0.04,0.02,0.02)$ & -0.044 \\
\hline 5 & $(0.06,0.09,0.09)$ & 0.058 & $(0.07,0.02,0.02)$ & 0.070 \\
\hline 6 & $(0.22,0.05,0.05)$ & 0.221 & $(0.17,0.01,0.01)$ & 0.171 \\
\hline 7 & $(-0.15,0.14,0.14)$ & -0.145 & $(-0.16,0.02,0.02)$ & -0.157 \\
\hline 8 & $(0.05,0.05,0.05)$ & 0.050 & $(-0.02,0.01,0.01)$ & -0.015 \\
\hline 9 & $(0.31,0.06,0.06)$ & 0.314 & $(0.31,0.01,0.01)$ & 0.309 \\
\hline 10 & $(-0.07,0.06,0.12)$ & -0.058 & $(-0.10,0.02,0.02)$ & -0.102 \\
\hline 11 & $(0.09,0.10,0.10)$ & 0.086 & $(0.11,0.02,0.02)$ & 0.106 \\
\hline 12 & $(0.00,0.11,0.11)$ & 0.000 & $(0.04,0.02,0.02)$ & 0.036 \\
\hline 13 & $(-0.05,0.11,0.11)$ & -0.048 & $(-0.05,0.02,0.02)$ & -0.052 \\
\hline 14 & $(-0.04,0.12,0.12)$ & -0.044 & $(-0.07,0.02,0.02)$ & -0.069 \\
\hline 15 & $(0.00,0.10,0.10)$ & -0.004 & $(0.00,0.02,0.02)$ & -0.003 \\
\hline 16 & $(-0.06,0.10,0.10)$ & -0.064 & $(-0.10,0.02,0.02)$ & -0.097 \\
\hline 17 & $(0.22,0.10,0.10)$ & 0.224 & $(0.30,0.02,0.02)$ & 0.300 \\
\hline 18 & $(-0.06,0.10,0.10)$ & -0.061 & $(-0.03,0.02,0.02)$ & -0.030 \\
\hline 19 & $(-0.08,0.10,0.10)$ & -0.079 & $(-0.04,0.02,0.02)$ & -0.043 \\
\hline 20 & $(-0.09,0.11,0.11)$ & -0.089 & $(-0.07,0.02,0.02)$ & -0.073 \\
\hline 21 & $(-0.18,0.10,0.10)$ & -0.177 & $(-0.12,0.02,0.02)$ & -0.116 \\
\hline
\end{tabular}


Table 10. Decreasing ordering of the importance of attributes

\begin{tabular}{|c|c|c|c|c|c|c|c|c|c|}
\hline \multirow{2}{*}{\multicolumn{2}{|c|}{$\begin{array}{c}\text { All hotels } \\
\text { Sym. Dr }\end{array}$}} & \multirow{2}{*}{\multicolumn{2}{|c|}{$\begin{array}{l}\text { Hotel } 1 \\
\text { Sym. Dr } \\
\end{array}$}} & \multirow{2}{*}{\multicolumn{2}{|c|}{$\begin{array}{l}\text { Hotel } 2 \\
\text { Sym. Dr } \\
\end{array}$}} & \multirow{2}{*}{\multicolumn{2}{|c|}{$\begin{array}{l}\text { Hotel } 3 \\
\text { Sym. Dr } \\
\end{array}$}} & \multicolumn{2}{|c|}{ Hotel 4} \\
\hline & & & & & & & & & \\
\hline 9 & 9 & 3 & 11 & 1 & 1 & 4 & 3 & 9 & 9 \\
\hline 4 & 2 & 11 & 3 & 2 & 3 & 5 & 21 & 17 & 17 \\
\hline 2 & 16 & 4 & 9 & 3 & 16 & 3 & 13 & 6 & 6 \\
\hline 8 & 6 & 9 & 8 & 10 & 12 & 21 & 2 & 11 & 11 \\
\hline 6 & 8 & 8 & 4 & 19 & 2 & 10 & 17 & 5 & 5 \\
\hline 10 & 14 & 19 & 14 & 16 & 14 & 8 & 5 & 8 & 12 \\
\hline 14 & 11 & 14 & 18 & 20 & 20 & 17 & 20 & 12 & 15 \\
\hline 16 & 17 & 18 & 17 & 8 & 19 & 13 & 8 & 15 & 8 \\
\hline 5 & 10 & 5 & 5 & 9 & 10 & 6 & 15 & 4 & 18 \\
\hline 3 & 4 & 17 & 19 & 14 & 4 & 14 & 10 & 2 & 19 \\
\hline 17 & 18 & 7 & 7 & 12 & 8 & 2 & 14 & 14 & 4 \\
\hline 18 & 3 & 2 & 10 & 4 & 7 & 1 & 1 & 13 & 2 \\
\hline 11 & 5 & 10 & 2 & 18 & 18 & 15 & 4 & 10 & 13 \\
\hline 1 & 1 & 16 & 12 & 7 & 15 & 20 & 6 & 18 & 14 \\
\hline 21 & 21 & 12 & 16 & 11 & 9 & 9 & 7 & 16 & 20 \\
\hline 13 & 13 & 6 & 6 & 13 & 6 & 7 & 16 & 19 & 16 \\
\hline 15 & 15 & 21 & 13 & 6 & 13 & 18 & 18 & 20 & 10 \\
\hline 7 & 7 & 13 & 21 & 5 & 5 & 11 & 9 & 3 & 21 \\
\hline 12 & 20 & 1 & 1 & 15 & 11 & 16 & 12 & 7 & 3 \\
\hline 19 & 12 & 15 & 15 & 17 & 17 & 12 & 11 & 1 & 7 \\
\hline 20 & 19 & 20 & 20 & 21 & 21 & 19 & 19 & 21 & 1 \\
\hline
\end{tabular}

Analyzing data on each hotel, we see that both approaches - symmetrical and drastic, in over half of the situations there are negative values. According to Ban et al. (2015a, 2015b), this may suggest an indirect relationship between the overall satisfaction and the perceived performance of attributes and a concentration of these attributes above a certain level does not lead to an increase in satisfaction but to its decrease. The position of the attribute "the hotel has sufficient catering facilities" is interesting, an attribute which is considered very important in all cases less than two (symmetrical and drastically) belonging to Hotel 4 .

\subsection{Segmented ordering of the importance of attributes}

The method summarized in Section 2 can be fructified in many directions. As example, in the present section we consider the division of the customers (respondents to the survey) according with certain criteria, apply the method and interpret the results.

\subsubsection{Experience with the hotel}

One of the question in the survey is related with the experience of the respondents: "Are you the first time at this hotel?", two answers ("yes" and "no") being possible. The results obtained by applying the proposed method are synthesized in Table 11 for all hotels and 
Table 11. Fuzzy importance and crisp importance of attributes related with the experience - All hotels

\begin{tabular}{|c|c|c|c|c|c|}
\hline \multicolumn{3}{|c|}{ "Yes" } & \multicolumn{3}{|c|}{ "No" } \\
\hline Attribute & Fuzzy importance & Importance & Attribute & Fuzzy importance & Importance \\
\hline 6 & $(0.56,0.04,0.04)$ & 0.563 & 16 & $(0.42,0.07,0.07)$ & 0.424 \\
\hline 4 & $(0.54,0.05,0.05)$ & 0.544 & 2 & $(0.37,0.11,0.11)$ & 0.370 \\
\hline 9 & $(0.54,0.07,0.07)$ & 0.537 & 10 & $(0.32,0.07,0.07)$ & 0.319 \\
\hline 14 & $(0.54,0.04,0.04)$ & 0.537 & 9 & $(0.31,0.06,0.06)$ & 0.306 \\
\hline 3 & $(0.51,0.04,0.04)$ & 0.511 & 8 & $(0.30,0.06,0.06)$ & 0.304 \\
\hline 8 & $(0.51,0.04,0.04)$ & 0.510 & 5 & $(0.30,0.05,0.05)$ & 0.296 \\
\hline 2 & $(0.46,0.05,0.05)$ & 0.455 & 18 & $(0.29,0.08,0.08)$ & 0.295 \\
\hline 11 & $(0.45,0.05,0.05)$ & 0.450 & 4 & $(0.22,0.08,0.08)$ & 0.220 \\
\hline 21 & $(0.43,0.05,0.05)$ & 0.427 & 17 & $(0.20,0.07,0.07)$ & 0.204 \\
\hline 1 & $(0.43,0.05,0.05)$ & 0.425 & 6 & $(0.19,0.07,0.07)$ & 0.192 \\
\hline 17 & $(0.42,0.04,0.04)$ & 0.417 & 11 & $(0.16,0.09,0.09)$ & 0.162 \\
\hline 10 & $(0.42,0.04,0.04)$ & 0.416 & 14 & $(0.13,0.07,0.07)$ & 0.126 \\
\hline 5 & $(0.39,0.04,0.04)$ & 0.394 & 3 & $(0.13,0.07,0.07)$ & 0.126 \\
\hline 13 & $(0.38,0.05,0.05)$ & 0.377 & 13 & $(0.12,0.07,0.07)$ & 0.116 \\
\hline 18 & $(0.36,0.04,0.04)$ & 0.365 & 1 & $(0.08,0.12,0.12)$ & 0.082 \\
\hline 7 & $(0.35,0.06,0.06)$ & 0.351 & 19 & $(0.07,0.10,0.10)$ & 0.070 \\
\hline 15 & $(0.33,0.05,0.05)$ & 0.325 & 15 & $(0.06,0.05,0.05)$ & 0.062 \\
\hline 16 & $(0.32,0.04,0.04)$ & 0.316 & 21 & $(0.05,0.07,0.07)$ & 0.053 \\
\hline 20 & $(0.30,0.05,0.05)$ & 0.300 & 7 & $(0.05,0.06,0.06)$ & 0.046 \\
\hline 12 & $(0.28,0.04,0.04)$ & 0.283 & 12 & $(0.04,0.06,0.06)$ & 0.043 \\
\hline 19 & $(0.25,0.05,0.05)$ & 0.254 & 20 & $(-0.04,0.08,0.08)$ & -0.039 \\
\hline
\end{tabular}

in Tables $12-15$ for each hotel, the rating of importance by triangular fuzzy numbers in Table 3 being considered.

The familiarity with a destination, given by the physical closeness, previous visits and knowledge about the destination play an important role in influencing individual perceptions about a destination (see Chi 2012; Hu, Ritchie 1993; Weaver et al. 2007), an aspect which may apply also to the previous experience with a museum (see Gil, Ritchie 2009) or a hotel. According to Alegre and Cladera (2006), the repetition of the visit has a limited impact on the overall satisfaction, therefore we should not see significant differences in the hierarchy of attributes.

The analysis of data overall and on each hotel shows hierarchies of the importance of attributes considerably changed, depending on the existence of a previous visit or not (see Table 16).

An interesting situation is held by the attributes "internet connection is available" and less "the staff is able to resolve the guests' problems" which in 8 of the 10 cases has approximately the same position regardless of a previous visit or not. The only exception is made by hotel 4 . These results confirm the validity of the proposed method, reinforcing the general perception of the constant importance that the internet connection has. 


\subsubsection{Motivation}

One of the question in the survey is related with the main reason of the travel: "What is the main reason why you are in this hotel?", seven answers being possible, but the most respondents were in categories M1 - "business" and M2 - "leisure". In the sequel we consider only the answers corresponding to these categories. The results obtained by applying the method in Section 2 to all hotels are synthesized in Table 17, the rating of importance by triangular fuzzy numbers in Table 3 being considered.

The relationship between satisfaction and the motivation of travel has been previously studied (see Meng et al. 2008), highlighting some notable distinctions between the business and leisure travel (see Kashyap, Bojanic 2000). In our study also, the hierarchy of the attributes by importance having as the respondent's motivation the business is radically different compared to the hierarchy of the attributes by importance having as the respondent's motivation the entertainment. In the case of tourism of business reasons, the first places are occupied by the following attributes: "the staff has an appropriate and professional look", "the room is clean enough", "the staff is able to provide information in a short time", while in the case of tourism motivated by entertainment on the top are the "friendliness of staff", "the safety of the installation of the hotel" and "the location of the hotel is suitable". The results are not surprising, they reflect the requirements of different categories of consumers and finding them in this study aligns the tourist behavior in Oradea with that of the tourists from other countries, similar results being obtained for business tourists in other studies (see Gundersen et al. 1996; Mccleary 1993). At the same time, the information processing method used is validated.

Table 12. Fuzzy importance and crisp importance of attributes related with the experience - Hotel 1

\begin{tabular}{cccccc}
\hline \multicolumn{5}{c}{ "Yes" } & \multicolumn{4}{c}{ "No" } \\
\hline Attribute & Fuzzy importance & Importance & Attribute & Fuzzy importance & Importance \\
\hline 9 & $(0.67,0.19,0.31)$ & 0.704 & 18 & $(0.33,0.16,0.33)$ & 0.368 \\
\hline 3 & $(0.67,0.23,0.31)$ & 0.694 & 11 & $(0.27,0.14,0.25)$ & 0.296 \\
\hline 14 & $(0.56,0.23,0.47)$ & 0.617 & 8 & $(0.16,0.16,0.29)$ & 0.192 \\
\hline 4 & $(0.56,0.22,0.28)$ & 0.573 & 17 & $(0.08,0.19,0.32)$ & 0.114 \\
\hline 8 & $(0.44,0.27,0.55)$ & 0.507 & 3 & $(0.06,0.19,0.31)$ & 0.085 \\
\hline 11 & $(0.46,0.14,0.21)$ & 0.474 & 5 & $(-0.03,0.20,0.36)$ & 0.014 \\
\hline 7 & $(0.28,0.23,0.47)$ & 0.338 & 2 & $(-0.08,0.30,0.60)$ & -0.003 \\
\hline 19 & $(0.31,0.13,0.14)$ & 0.317 & 9 & $(-0.07,0.17,0.30)$ & -0.041 \\
\hline 5 & $(0.23,0.14,0.15)$ & 0.232 & 12 & $(-0.10,0.17,0.29)$ & -0.067 \\
\hline 17 & $(0.17,0.21,0.42)$ & 0.221 & 16 & $(-0.13,0.24,0.49)$ & -0.072 \\
\hline 13 & $(0.11,0.29,0.55)$ & 0.175 & 19 & $(-0.09,0.22,0.31)$ & -0.074 \\
\hline 10 & $(0.11,0.29,0.55)$ & 0.175 & 6 & $(-0.16,0.23,0.45)$ & -0.101 \\
\hline
\end{tabular}


End of Table 12

\begin{tabular}{cccccc}
\hline \multicolumn{2}{c}{ "Yes" } & \multicolumn{2}{c}{ "No" } \\
\hline Attribute & Fuzzy importance & Importance & Attribute & Fuzzy importance & Importance \\
\hline 18 & $(0.00,0.23,0.47)$ & 0.058 & 1 & $(-0.18,0.21,0.42)$ & -0.129 \\
\hline 15 & $(-0.07,0.19,0.37)$ & -0.028 & 10 & $(-0.18,0.21,0.42)$ & -0.129 \\
\hline 20 & $(-0.08,0.21,0.42)$ & -0.032 & 4 & $(-0.17,0.15,0.30)$ & -0.132 \\
\hline 12 & $(-0.16,0.20,0.40)$ & -0.109 & 14 & $(-0.20,0.20,0.40)$ & -0.156 \\
\hline 2 & $(-0.14,1.44,1.44)$ & -0.144 & 7 & $(-0.21,0.15,0.26)$ & -0.178 \\
\hline 16 & $(-0.14,1.44,1.44)$ & -0.144 & 21 & $(-0.22,0.13,0.25)$ & -0.186 \\
\hline 6 & $(-0.14,1.44,1.44)$ & -0.144 & 13 & $(-0.29,0.17,0.29)$ & -0.262 \\
\hline 21 & $(-0.14,1.44,1.44)$ & -0.144 & 20 & $(-0.31,0.15,0.27)$ & -0.278 \\
\hline 1 & $(-0.28,0.23,0.47)$ & -0.221 & 15 & $(-0.37,0.18,0.24)$ & -0.351 \\
\hline
\end{tabular}

Table 13. Fuzzy importance and crisp importance of attributes related with the experience - Hotel 2

\begin{tabular}{cccccc}
\hline \multicolumn{2}{c}{ "Yes" } & & & "No" & \\
\hline Attribute & Fuzzy importance & Importance & Attribute & Fuzzy importance & Importance \\
\hline 14 & $(1.00,1.00,1.00)$ & 1.000 & 1 & $(0.89,0.47,0.47)$ & 0.886 \\
\hline 16 & $(-0.11,0.50,1.00)$ & 0.014 & 2 & $(0.76,0.41,0.54)$ & 0.791 \\
\hline 11 & $(-0.11,0.50,1.00)$ & 0.014 & 10 & $(0.76,0.41,0.54)$ & 0.791 \\
\hline 4 & $(-0.11,0.50,1.00)$ & 0.014 & 19 & $(0.76,0.41,0.54)$ & 0.791 \\
\hline 10 & $(-0.11,0.50,1.00)$ & 0.014 & 3 & $(0.72,0.24,0.51)$ & 0.789 \\
\hline 18 & $(-0.11,0.50,1.00)$ & 0.014 & 16 & $(0.67,0.20,0.47)$ & 0.736 \\
\hline 8 & $(-0.11,0.50,1.00)$ & 0.014 & 9 & $(0.67,0.38,0.47)$ & 0.691 \\
\hline 19 & $(-0.11,0.50,1.00)$ & 0.014 & 8 & $(0.67,0.38,0.47)$ & 0.691 \\
\hline 15 & $(-0.17,0.38,0.75)$ & -0.073 & 18 & $(0.56,0.31,0.56)$ & 0.617 \\
\hline 9 & $(-0.17,0.38,0.75)$ & -0.073 & 20 & $(0.56,0.31,0.56)$ & 0.617 \\
\hline 12 & $(-0.11,1.05,1.05)$ & -0.105 & 4 & $(0.48,0.16,0.34)$ & 0.529 \\
\hline 13 & $(-0.11,1.05,1.05)$ & -0.105 & 12 & $(0.44,0.38,0.38)$ & 0.438 \\
\hline 7 & $(-0.11,1.05,1.05)$ & -0.105 & 7 & $(0.39,0.24,0.28)$ & 0.400 \\
\hline 2 & $(-0.11,1.05,1.05)$ & -0.105 & 11 & $(0.39,0.24,0.28)$ & 0.400 \\
\hline 3 & $(-0.11,1.05,1.05)$ & -0.105 & 5 & $(0.36,0.25,0.25)$ & 0.358 \\
\hline 1 & $(-0.11,1.05,1.05)$ & -0.105 & 15 & $(0.31,0.33,0.33)$ & 0.307 \\
\hline 6 & $(-0.11,1.05,1.05)$ & -0.105 & 17 & $(0.17,0.33,0.66)$ & 0.256 \\
\hline 20 & $(-0.11,1.05,1.05)$ & -0.105 & 14 & $(0.17,0.33,0.66)$ & 0.256 \\
\hline 21 & $(-0.11,1.05,1.05)$ & -0.105 & 6 & $(0.17,0.33,0.66)$ & 0.256 \\
\hline 5 & $(-0.22,0.33,0.65)$ & -0.136 & 13 & $(0.17,0.33,0.66)$ & 0.256 \\
\hline 17 & $(-0.22,0.33,0.65)$ & -0.136 & 21 & $(-0.14,0.47,0.89)$ & -0.035 \\
\hline & & & & & \\
\hline
\end{tabular}


Table 14. Fuzzy importance and crisp importance of attributes related with the experience - Hotel 3

\begin{tabular}{|c|c|c|c|c|c|}
\hline \multicolumn{3}{|c|}{ "Yes" } & \multicolumn{3}{|c|}{ "No" } \\
\hline Attribute & Fuzzy importance & Importance & Attribute & Fuzzy importance & Importance \\
\hline 2 & $(0.64,0.21,0.21)$ & 0.638 & 5 & $(0.43,0.24,0.25)$ & 0.430 \\
\hline 3 & $(0.59,0.14,0.16)$ & 0.598 & 21 & $(0.16,0.24,0.24)$ & 0.160 \\
\hline 4 & $(0.59,0.16,0.16)$ & 0.595 & 4 & $(0.16,0.24,0.24)$ & 0.156 \\
\hline 1 & $(0.51,0.21,0.21)$ & 0.509 & 16 & $(0.05,0.66,0.66)$ & 0.047 \\
\hline 21 & $(0.46,0.18,0.18)$ & 0.465 & 15 & $(0.02,0.28,0.28)$ & 0.020 \\
\hline 14 & $(0.45,0.16,0.16)$ & 0.453 & 8 & $(0.00,0.21,0.21)$ & 0.000 \\
\hline 6 & $(0.44,0.20,0.20)$ & 0.440 & 10 & $(0.00,0.29,0.29)$ & 0.000 \\
\hline 8 & $(0.44,0.20,0.20)$ & 0.440 & 13 & $(0.00,0.26,0.26)$ & 0.000 \\
\hline 7 & $(0.42,0.14,0.14)$ & 0.417 & 17 & $(-0.03,0.24,0.24)$ & -0.032 \\
\hline 10 & $(0.41,0.16,0.16)$ & 0.411 & 3 & $(-0.05,0.29,0.29)$ & -0.054 \\
\hline 17 & $(0.41,0.19,0.19)$ & 0.409 & 18 & $(-0.07,0.23,0.23)$ & -0.069 \\
\hline 13 & $(0.39,0.26,0.26)$ & 0.390 & 6 & $(-0.21,0.24,0.24)$ & -0.205 \\
\hline 5 & $(0.39,0.17,0.17)$ & 0.387 & 14 & $(-0.21,0.22,0.22)$ & -0.207 \\
\hline 20 & $(0.28,0.14,0.14)$ & 0.281 & 20 & $(-0.24,0.28,0.28)$ & -0.243 \\
\hline 11 & $(0.28,0.22,0.22)$ & 0.278 & 9 & $(-0.24,0.28,0.28)$ & -0.243 \\
\hline 18 & $(0.22,0.22,0.22)$ & 0.225 & 11 & $(-0.34,0.34,0.34)$ & -0.338 \\
\hline 9 & $(0.21,0.22,0.22)$ & 0.205 & 19 & $(-0.38,0.38,0.38)$ & -0.377 \\
\hline 12 & $(0.19,0.19,0.19)$ & 0.190 & 2 & $(-0.45,0.45,0.45)$ & -0.453 \\
\hline 15 & $(0.08,0.24,0.24)$ & 0.080 & 1 & $(-0.45,0.45,0.45)$ & -0.454 \\
\hline 19 & $(-0.01,0.26,0.26)$ & -0.009 & 12 & $(-0.45,0.26,0.25)$ & -0.456 \\
\hline 16 & $(-0.10,0.25,0.25)$ & -0.101 & 7 & $(-0.48,0.28,0.26)$ & -0.483 \\
\hline
\end{tabular}

Table 15. Fuzzy importance and crisp importance of attributes related with the experience - Hotel 4

\begin{tabular}{cccccc}
\hline \multicolumn{5}{c}{ "Yes" } & \multicolumn{4}{c}{ "No" } \\
\hline Attribute & Fuzzy importance & Importance & Attribute & Fuzzy importance & Importance \\
\hline 6 & $(0.51,0.10,0.10)$ & 0.508 & 17 & $(0.24,0.14,0.27)$ & 0.270 \\
\hline 9 & $(0.40,0.12,0.12)$ & 0.397 & 9 & $(0.24,0.13,0.13)$ & 0.236 \\
\hline 8 & $(0.22,0.10,0.10)$ & 0.215 & 2 & $(0.09,0.14,0.18)$ & 0.103 \\
\hline 17 & $(0.20,0.15,0.15)$ & 0.204 & 11 & $(0.05,0.10,0.10)$ & 0.046 \\
\hline 4 & $(0.17,0.10,0.19)$ & 0.190 & 5 & $(0.02,0.12,0.20)$ & 0.038 \\
\hline 14 & $(0.10,0.18,0.18)$ & 0.102 & 16 & $(0.04,0.13,0.13)$ & 0.035 \\
\hline 15 & $(0.10,0.16,0.16)$ & 0.098 & 12 & $(-0.06,0.14,0.28)$ & -0.023 \\
\hline 11 & $(0.10,0.16,0.16)$ & 0.098 & 13 & $(-0.07,0.14,0.14)$ & -0.070 \\
\hline 5 & $(0.09,0.15,0.15)$ & 0.087 & 15 & $(-0.16,0.14,0.27)$ & -0.131 \\
\hline 12 & $(0.06,0.16,0.16)$ & 0.064 & 6 & $(-0.15,0.10,0.10)$ & -0.149 \\
\hline 18 & $(0.06,0.16,0.16)$ & 0.058 & 7 & $(-0.17,0.14,0.18)$ & -0.156 \\
\hline
\end{tabular}


End of Table 15

\begin{tabular}{cccccc}
\hline & "Yes" & & \multicolumn{2}{c}{ "No" } \\
\hline Attribute & Fuzzy importance & Importance & Attribute & Fuzzy importance & Importance \\
\hline 20 & $(0.02,0.16,0.16)$ & 0.017 & 10 & $(-0.17,0.13,0.14)$ & -0.166 \\
\hline 3 & $(-0.02,0.10,0.19)$ & 0.000 & 8 & $(-0.20,0.16,0.16)$ & -0.203 \\
\hline 1 & $(-0.03,0.10,0.20)$ & -0.008 & 19 & $(-0.24,0.15,0.30)$ & -0.203 \\
\hline 13 & $(-0.02,0.16,0.16)$ & -0.019 & 18 & $(-0.25,0.14,0.28)$ & -0.220 \\
\hline 19 & $(-0.04,0.18,0.18)$ & -0.036 & 21 & $(-0.26,0.10,0.19)$ & -0.233 \\
\hline 21 & $(-0.07,0.12,0.22)$ & -0.045 & 20 & $(-0.31,0.14,0.14)$ & -0.310 \\
\hline 10 & $(-0.09,0.12,0.22)$ & -0.071 & 4 & $(-0.31,0.14,0.14)$ & -0.310 \\
\hline 7 & $(-0.14,0.21,0.21)$ & -0.141 & 3 & $(-0.31,0.14,0.14)$ & -0.310 \\
\hline 2 & $(-0.17,0.10,0.19)$ & -0.145 & 14 & $(-0.35,0.18,0.18)$ & -0.350 \\
\hline 16 & $(-0.15,0.15,0.15)$ & -0.149 & 1 & $(-0.42,0.13,0.13)$ & -0.417 \\
\hline
\end{tabular}

Table 16. Decreasing ordering of the importance of attributes related with the experience

\begin{tabular}{|c|c|c|c|c|c|c|c|c|c|}
\hline \multicolumn{2}{|c|}{ All hotels } & \multicolumn{2}{|c|}{ Hotel 1} & \multicolumn{2}{|c|}{ Hotel 2} & \multicolumn{2}{|c|}{ Hotel 3} & \multicolumn{2}{|c|}{ Hotel 4} \\
\hline "Yes" & "No" & "Yes" & "No" & "Yes" & "No" & "Yes" & "No" & "Yes" & "No" \\
\hline 6 & 16 & 9 & 18 & 14 & 1 & 2 & 16 & 6 & 17 \\
\hline 4 & 2 & 3 & 11 & 2 & 2 & 3 & 5 & 9 & 9 \\
\hline 9 & 10 & 14 & 8 & 3 & 10 & 4 & 21 & 8 & 2 \\
\hline 14 & 9 & 4 & 17 & 6 & 19 & 1 & 4 & 17 & 11 \\
\hline 3 & 8 & 8 & 3 & 7 & 3 & 21 & 15 & 4 & 5 \\
\hline 8 & 5 & 11 & 5 & 12 & 16 & 14 & 8 & 14 & 16 \\
\hline 2 & 18 & 7 & 2 & 13 & 9 & 6 & 10 & 15 & 12 \\
\hline 11 & 4 & 19 & 9 & 1 & 8 & 8 & 13 & 11 & 13 \\
\hline 21 & 17 & 5 & 12 & 20 & 18 & 7 & 17 & 5 & 15 \\
\hline 1 & 6 & 17 & 16 & 21 & 20 & 10 & 3 & 12 & 6 \\
\hline 17 & 11 & 13 & 19 & 8 & 4 & 17 & 18 & 18 & 7 \\
\hline 10 & 14 & 10 & 6 & 19 & 12 & 13 & 6 & 20 & 10 \\
\hline 5 & 3 & 18 & 1 & 16 & 11 & 5 & 14 & 3 & 8 \\
\hline 13 & 13 & 6 & 10 & 18 & 7 & 20 & 20 & 1 & 19 \\
\hline 18 & 1 & 2 & 4 & 10 & 5 & 11 & 9 & 13 & 18 \\
\hline 7 & 19 & 16 & 14 & 11 & 15 & 18 & 11 & 19 & 21 \\
\hline 15 & 15 & 21 & 7 & 4 & 17 & 9 & 19 & 21 & 20 \\
\hline 16 & 21 & 15 & 21 & 15 & 14 & 12 & 2 & 10 & 4 \\
\hline 20 & 7 & 20 & 13 & 9 & 6 & 15 & 12 & 7 & 3 \\
\hline 12 & 12 & 12 & 20 & 5 & 13 & 19 & 1 & 2 & 14 \\
\hline 19 & 20 & 1 & 15 & 17 & 21 & 16 & 7 & 16 & 1 \\
\hline
\end{tabular}


Table 17. Fuzzy importance and crisp importance of attributes related with the motivation - All hotels

\begin{tabular}{|c|c|c|c|c|c|}
\hline \multicolumn{3}{|c|}{ M1 } & \multicolumn{3}{|c|}{ M2 } \\
\hline Attribute & Fuzzy importance & Importance & Attribute & Fuzzy importance & Importance \\
\hline 4 & $(0.56,0.06,0.07)$ & 0.562 & 16 & $(0.69,0.29,0.69)$ & 0.794 \\
\hline 2 & $(0.53,0.06,0.06)$ & 0.533 & 13 & $(0.53,0.26,0.53)$ & 0.595 \\
\hline 9 & $(0.50,0.05,0.05)$ & 0.496 & 5 & $(0.53,0.26,0.53)$ & 0.595 \\
\hline 1 & $(0.49,0.06,0.06)$ & 0.495 & 20 & $(0.50,0.25,0.50)$ & 0.563 \\
\hline 6 & $(0.48,0.05,0.05)$ & 0.479 & 21 & $(0.41,0.28,0.55)$ & 0.477 \\
\hline 5 & $(0.47,0.04,0.04)$ & 0.473 & 18 & $(0.37,0.24,0.48)$ & 0.428 \\
\hline 10 & $(0.46,0.05,0.05)$ & 0.463 & 17 & $(0.29,0.25,0.49)$ & 0.353 \\
\hline 8 & $(0.45,0.05,0.05)$ & 0.445 & 4 & $(0.29,0.29,0.29)$ & 0.291 \\
\hline 14 & $(0.42,0.06,0.06)$ & 0.420 & 6 & $(0.26,0.39,0.43)$ & 0.269 \\
\hline 16 & $(0.37,0.05,0.05)$ & 0.372 & 15 & $(0.20,0.25,0.40)$ & 0.238 \\
\hline 3 & $(0.32,0.05,0.05)$ & 0.318 & 14 & $(0.16,0.32,0.40)$ & 0.178 \\
\hline 18 & $(0.32,0.05,0.05)$ & 0.317 & 9 & $(0.06,0.29,0.55)$ & 0.124 \\
\hline 11 & $(0.29,0.05,0.05)$ & 0.288 & 3 & $(0.06,0.29,0.55)$ & 0.124 \\
\hline 17 & $(0.26,0.05,0.05)$ & 0.263 & 10 & $(0.06,0.29,0.55)$ & 0.124 \\
\hline 21 & $(0.25,0.07,0.07)$ & 0.246 & 2 & $(0.00,0.32,0.65)$ & 0.081 \\
\hline 19 & $(0.21,0.06,0.06)$ & 0.214 & 11 & $(0.00,0.32,0.65)$ & 0.081 \\
\hline 12 & $(0.18,0.05,0.05)$ & 0.185 & 7 & $(-0.19,0.33,0.66)$ & -0.106 \\
\hline 15 & $(0.16,0.05,0.05)$ & 0.163 & 1 & $(-0.26,0.32,0.65)$ & -0.178 \\
\hline 7 & $(0.15,0.05,0.05)$ & 0.151 & 8 & $(-0.26,0.26,0.43)$ & -0.215 \\
\hline 13 & $(0.11,0.05,0.05)$ & 0.107 & 19 & $(-0.30,0.31,0.63)$ & -0.224 \\
\hline 20 & $(-0.05,0.07,0.07)$ & -0.045 & 12 & $(-0.35,0.35,0.52)$ & -0.303 \\
\hline
\end{tabular}

\section{Conclusions}

With respect to the hypotheses $\mathrm{H} 1-\mathrm{H} 3$ in Introduction we can formulate the following conclusions:

H1: The differences between the hierarchy of attributes obtained considering a symmetrical scale and the hierarchy of the attributes resulted considering a drastic scale is not that important as it is suggested in the literature, therefore the hypothesis is not confirmed.

$\mathrm{H} 2$ : The hypothesis is confirmed, that is the analysis of data overall and on each hotel shows hierarchies of the importance of attributes considerably changed, depending on the existence of a previous visit or not.

H3: The hierarchy of the attributes by importance having as the respondent's motivation the business is radically different compared to the hierarchy of the attributes by importance having as the respondent's motivation the entertainment, therefore the hypothesis is confirmed. 


\section{References}

Abalo, J.; Varela, J.; Manzano, V. 2007. Importance values for Importance-Performance Analysis: a formula for spreading out values derived from preference rankings, Journal of Bussiness Research 60(2): 115-121. http://dx.doi.org/10.1016/j.jbusres.2006.10.009

Alegre, J.; Cladera, M. 2006. Repeat visitation in mature sun and sand holiday destinations, Journal of Travel Research 44(3): 288-297. http://dx.doi.org/10.1177/0047287505279005

Bacon, D. R. 2003. A comparison of approaches to Importance-Performance Analysis, International Journal of Marketing Research 45: 55-71.

Ban, O. I. 2011. Fuzzy multicriteria decision making method applied to selection of the best touristic destinations, International Journal of Mathematical Models and Methods in Applied Science 5: 264-271.

Ban, A. I.; Ban, O. I. 2012. Optimization and extensions of a fuzzy multicriteria decision making method and applications to selection of touristic destinations, Expert Systems with Applications 39(8): 7216-7225. http://dx.doi.org/10.1016/j.eswa.2012.01.055

Ban, A. I.; Ban, O. I.; Tușe, D. A. 2015a. Calculation of the fuzzy importance of attributes based on the correlation coefficient, applied to the quality of hotel services, Journal of Intelligent and Fuzzy Systems 30(1): 583-596. http://dx.doi.org/10.3233/IFS-151840

Ban, A. I.; Ban, O. I.; Tușe, D. A. 2015b. Derived fuzzy importance of attributes based on the weakest triangular norm-based fuzzy arithmetic and applications to the hotel services, submitted.

Ban, A. I.; Coroianu, L. 2015. Simplifying the search for effective ranking of fuzzy numbers, IEEE Transactions on Fuzzy Systems 23(2): 327-339. http://dx.doi.org/10.1109/TFUZZ.2014.2312204

Ban, O. I.; Meșter, I. T. 2014. Using Kano two dimensional service quality classification and characteristic analysis from the perspective of hotels' clients of Oradea, Journal of Tourism-Studies and Research in Tourism 18: 30-36.

Chanas, S. 2001. On the interval approximation of a fuzzy number, Fuzzy Sets and Systems 122(2): 353-356. http://dx.doi.org/10.1016/S0165-0114(00)00080-4

Chi, C. G. 2012. An examination of destination loyalty: differences between first-time and repeat visitors, Journal of Hospitality \& Tourism Research 36(1): 3-24. http://dx.doi.org/10.1177/1096348010382235

Chien, C. J.; Tsai, H. H. 2000. Using fuzzy numbers to evaluate perceived service quality, Fuzzy Sets and Systems 116(2): 289-300. http://dx.doi.org/10.1016/S0165-0114(98)00239-5

Chu, T.-C.; Lin, Y. 2009. An extension to fuzzy MCDM, Computers and Mathematics with Applications 57(3): 445-454. http://dx.doi.org/10.1016/j.camwa.2008.10.076

Deng, W. 2007. Using a revised importance-performance analysis approach: the case of Taiwanese hot springs tourism, Tourism Management 28(5): 1274-1284.

http://dx.doi.org/10.1016/j.tourman.2006.07.010

Deng, W. J. 2008. Fuzzy importance-performance analysis for determining critical service attributes, International Journal of Service Industry Management 19(2): 252-270. http://dx.doi.org/10.1108/09564230810869766

Deng, W. J.; Pei, W. 2009. Fuzzy neural based importance-performance analysis for determining critical service attributes, Expert Systems with Applications 36(2): 3774-3784. http://dx.doi.org/10.1016/j.eswa.2008.02.063

Diamond, P.; Kloeden, P. 1994. Metric spaces of fuzzy sets. Theory and applications. Singapore: World Scientific. http://dx.doi.org/10.1142/2326

Dolnicar, S. 2007. Accepted standards undermining the validity of tourism research, in A. G. Woodside (Ed.). Advances in culture, tourism and hospitality research, Vol. 1. Elsevier, 131-181.

http://dx.doi.org/10.1016/S1871-3173(06)01005-6 
Dubois, D.; Prade, H. 1978. Operations on fuzzy numbers, International Journal of Systems Sciences 9(6): 613-626. http://dx.doi.org/10.1080/00207727808941724

Dubois, D.; Prade, H. 1987. The mean value of a fuzzy number, Fuzzy Sets and Systems 24(3): 279-300. http://dx.doi.org/10.1016/0165-0114(87)90028-5

Feng, M.; Mangan, J.; Wong, C.; Xu, M.; Lalwani, C. 2014. Investigating the different approaches to importance-performance analysis, The Service Industries Journal 34(12): 1021-1041. http://dx.doi.org/10.1080/02642069.2014.915949

Gil, S. M.; Ritchie, J. R. B. 2009. Understanding the museum image formation process: a comparison of residents and tourists, Journal of Travel Research 47: 480-493.

Gundersen, M. G.; Heide, M.; Uff, H. 1996. Hotel guest satisfaction among business travelers: what are the important factors?, Olsson Cornell Hotel and Restaurant Administration Quarterly 37(2): 72-81. http://dx.doi.org/10.1016/0010-8804(96)83104-1

Hair, J. F.; Anderson, R. E.; Tatham, R. L.; Black, W. C. 1995. Multivariate data analysis. New Jersey: Pretince-Hall, Upper Saddle River.

Hancock, G. R.; Klockars, A. J. 1991. The effect of scale manipulations on validity: Targeting frequency rating scales for anticipated performance levels, Applied Ergonomics 22(3): 147-154.

http://dx.doi.org/10.1016/0003-6870(91)90153-9

Hong, D. H. 2006. Fuzzy measures for a correlation coefficient of fuzzy numbers under $\mathrm{T}_{\mathrm{W}}$ (the weakest $\mathrm{t}$-norm)-based fuzzy arithmetic operations, Information Sciences 176(2): 150-160. http://dx.doi.org/10.1016/j.ins.2004.11.005

Hong, D. H. 2001. Shape preserving multiplications of fuzzy intervals, Fuzzy Sets and Systems 123(1): 81-84. http://dx.doi.org/10.1016/S0165-0114(00)00107-X

Hong, D. H. 2002. On shape-preserving additions of fuzzy intervals, Journal of Mathematical Analysis and Applications 267(1): 369-376. http://dx.doi.org/10.1006/jmaa.2001.7788

Hu, Y.; Ritchie, J. R. B. 1993. Measuring destination attractiveness: a contextual approach, Journal of Travel Research 32(2): 25-34. http://dx.doi.org/10.1177/004728759303200204

Kashyap, R.; Bojanic, D. C. 2000. A structural analysis of value, quality and price perceptions of business and leisure travelers, Journal of Travel Research 39(1): 45-51.

http://dx.doi.org/10.1177/004728750003900106

Liu, S.-T.; Kao, C. 2002. Fuzzy measures for correlation coeffcient of fuzzy numbers, Fuzzy Sets and Systems 128(2): 267-275. http://dx.doi.org/10.1016/S0165-0114(01)00199-3

Matzler, K.; Sauerwein, E.; Heischmidt, K.A. 2003. Importance-performance analysis revisited: the role of the factor structure of customer satisfaction, The Service Industries Journal 23(2): 112-129. http://dx.doi.org/10.1080/02642060412331300912

Mccleary, K. W.; Weaver, P. A.; Hutchinson, J. C. 1993. Hotel selection factors as they relate to business travel situations, Journal of Travel Research 32(2): 42-48. http://dx.doi.org/10.1177/004728759303200206

Meng, F.; Tepanon, Y.; Uysal, M. 2008. Measuring tourist satisfaction by attribute and motivation: the case of a nature-based resort, Journal of Vacation Marketing 14(1): 41-56. http://dx.doi.org/10.1177/1356766707084218

Mount, D. J.; Sciarini, M. P. 1999. IPA and DSI: enhancing the usefulness of student evaluation of teaching data, Journal of Hospitality \& Tourism Education 10(4): 8-14.

http://dx.doi.org/10.1080/10963758.1999.10685203

Mount, D. J. 2005. An empirical application of quantitative derived importance-performance analysis (QDIPA) for employee satisfaction, Journal of Quality Assurance in Hospitality \& Tourism 6(1-2): 65-76. http://dx.doi.org/10.1300/J162v06n01_05 
Preston, C. C.; Colman, A. M. 2000. Optimal number of response categories in rating scales: reliability, validity, discriminating power, and respondent preferences, Acta Psychologica 104(1): 1-15. http://dx.doi.org/10.1016/S0001-6918(99)00050-5

Snedecor, G. W.; Cochran, W. G. 1967. Statistical methods. Iowa: Iowna State University Press.

Stanojević, B.; Dzițac, I.; Dzițac, S. 2015. On the ratio of fuzzy numbers-exact membership function computation and applications to decision making, Technological and Economic Development of Economy 21(5): 815-832. http://dx.doi.org/10.3846/20294913.2015.1093563

Watson, D. 1992. Correcting for acquiescent response bias in the absence of a balanced scale: an application to class consciousness, Sociological Methods and Research 21(1): 52-88. http://dx.doi.org/10.1177/0049124192021001003

Weaver, P. A.; Weber, K.; McCleary, K. W. 2007. Destination evaluation: the role of previous travel experience and trip characteristics, Journal of Travel Research 45(3): 333-344. http://dx.doi.org/10.1177/0047287506292702

Wei, G. W. 2011. FIOWHM operator and its application to multiple attribute group decision making, Expert Systems with Applications 38(4): 2984-2989. http://dx.doi.org/10.1016/j.eswa.2010.08.087

Wei, G. W.; Zhao, X. F.; Lin, R.; Wang, H. J. 2012. Generalized triangular fuzzy correlated averaging operator and their application to multiple attribute decision making, Applied Mathematical Modelling 36(7): 2975-2982. http://dx.doi.org/10.1016/j.apm.2011.09.062

Wei, G. W.; Zhao, X. F.; Wang, H. J.; Lin, R. 2013. Fuzzy power aggregation operators and their application to multiple attribute group decision making, Technological and Economic Development of Economy 19(3): 377-396. http://dx.doi.org/10.3846/20294913.2013.821684

Wu, W. Y.; Hsiao, S. W.; Kuo, H. P. 2004. Fuzzy set theory based decision model for determining market position and developing strategy for hospital service quality, Total Quality Management 15(4): 439-456. http://dx.doi.org/10.1080/1478336042000183587

Yeh, C.-H.; Kuo, Y.-L. 2003. Evaluating passenger services of Asia-Pacific international airports, Transportation Research Part E 39(1): 35-48. http://dx.doi.org/10.1016/S1366-5545(02)00017-0

Zadeh, L. A. 1965. Fuzzy Sets, Information and Control 8(3): 338-353. http://dx.doi.org/10.1016/S0019-9958(65)90241-X

Zadeh, L. A. 1978. Fuzzy sets as a basis for a theory of possibility, Fuzzy Sets and Systems 1(1): 3-28. http://dx.doi.org/10.1016/0165-0114(78)90029-5

Zhao, X. F.; Lin, R.; Wei, G. W. 2013. Fuzzy prioritized operators and their application to multiple attribute group decision making, Applied Mathematical Modelling 37(7): 4759-4770.

http://dx.doi.org/10.1016/j.apm.2012.09.048

Olimpia-Iuliana BAN. Doctor, Associate Professor. Department of Economics, Faculty of Economic Sciences, University of Oradea, Romania. She is PhD in Economics (Marketing), from 2005. She is a member of AGER (General Association of Romanian Economists) and member of AROMAR (Romanian Marketing Association). She has been Head of Department of Economics between 2008-2011 and 2012-2014. Author of about 80 scientific publications. Research interests: tourism, marketing, marketing research, tourism promotion.

Ioan Gheorghe TARA. Doctor. Tara is a PhD professor at the University of Oradea, Faculty of Economic Sciences, Department of Finance and Accounting. He is a certified accountant, member of some professional organizations in Romania. ACCA from United Kingdom acknowledged him as an expert in international standards of financial audit. He published more than 80 studies in the field. 
Victoria BOGDAN. Doctor. She is $\mathrm{PhD}$ in Economics (Accounting) from 2004. She is associate professor, Department of Finance \& Accounting, Faculty of Economic Sciences, University of Oradea, Romania. She is a cerfied accountant and financial auditor member of the following professional organisations: CECCAR (Certfified Accontants Association from Romania), CAFR (Chamber of Romanian Financial Auditors) and AGER (General Association of Romanian Economists). Author of about 50 scientific articles and 10 books. Research interests: accounting, IFRS, behavioral finance and accounting, financial reporting, audit, professional judgment.

Delia TUȘE. PhD Candidate, Assistant Lecturer. Department of Mathematics and Informatics, Science Faculty, University of Oradea. First degree in mathematics and informatics, University of Oradea (2003). Master in real and complex analysis (2005). PhD Candidate (present). Programmer Analyst to 3 companies from Oradea, Romania (2001-2007). Trainer IT to IT Services project (2008-2009). Author of about 7 scientific articles. Research interests: decision theory, multiple criteria decision making and fuzzy set theory.

Simona Gabriela BOLOGA. Doctor. Bologa Gabriela, obtained her doctoral degree in Management in 2010 from the West University of Timisoara. She is Jean Monet Professor staring with 2007 and now she is the Dean of the Economics Faculty, Agora University, Oradea, being in charge of Financial Analysis and Project Management research and studies. She authored and co-authored more than 45 scientific papers in the field of the above subjects. Her research interests include different aspects of economical and financial studies. 